\title{
The influence of breast skin thickness variation with aging on the mammographic $X$-ray spectra: A Monte Carlo study
}

\author{
M. Zadehrafi* \\ Horia Hulubei National Institute for Research and Development in Physics and Nuclear Engineering, 30 Reactorului \\ Street, P.O. Box MG-6, RO-077125 Bucharest-Magurele, Romania
}

\begin{abstract}
- Original article

\section{*Corresponding author: \\ Mastaneh Zadehrafi, Ph.D., \\ E-mail:}

mastaneh.zadehrafi@nipne.ro

Revised: April 2021

Accepted: June 2021

Int. J. Radiat. Res., October 2021; 19(4): $971-978$

DOI: $10.29242 /$ ijrr.19.4.971

Background: To approach the mammographic X-ray investigations in a metrological manner, any potential improvement needs to be considered. In this study, the influence of skin thickness variation on the mammographic $X$ ray spectra was investigated. Additionally, some attenuation parameters such as linear and mass attenuation coefficients, mean free path, and half-value layer thickness were assessed for exposure of the skin to the mammographic $\mathrm{X}$-rays. Materials and Methods: A theoretical experiment was performed using MCNP6.2 by simulation of two collimators, a detection area, a skin sample, and a point monoenergetic X-ray source. Carrying out this experiment, the above-mentioned parameters were estimated. As a validation process, the mass attenuation coefficients obtained by MCNP were compared with the results of XCOM. Results: The good agreement between the results of MCNP and XCOM reveals that the geometrical setup was modeled correctly by MCNP. It also implies the reliability of the Monte Carlo technique for attenuation calculations of low-energy X-rays through the skin. Upon increasing the breast skin thickness, its transmission factor against mammographic $X$-rays decreases linearly, and it increases by increasing the photon energy. The older the patient, the more transmission factor of the breast skin against the low energy $\mathrm{X}$-ray. Conclusion: Increasing the $\mathrm{X}$-ray energy leads to less sensitivity of the transmission factor to the skin thickness. Thus, to have the best possible quality of X-ray mammography images, the patient's age may also be considered as well as the other factors, when selecting the proper energies and for fine-tuning the used $\mathrm{X}$-ray generators.
\end{abstract}

Keywords: X-ray, mammography, breast skin, attenuation coefficients, transmission factor, Monte Carlo, MCNP.

\section{INTRODUCTION}

Breast cancer is one of the most common cancers diagnosed in women. Mammography is a specific type of breast imaging that uses low-energy X-rays to look for early signs of breast cancer. The X-ray picture of the breast is called a mammogram. Since many pathological conditions, especially cancer, produce very small physical changes that might be difficult to be visualized by X-ray imaging, the mammography examination requires the highest image quality of all the X-ray procedures (1).

Obviously, the outermost layer of the breast exposed to X-ray diagnostic equipment is the skin. On the other hand, the human skin shows some alterations with advancing age, which the thickness variation is one of them. In 2016, Coltman et al. (2) performed a study regarding the age-related changes to the female breast skin by measuring the breast skin thickness of 339 women in four age groups. The results of the study revealed that breast skin thickness significantly decreases from 45 years of age 
onwards.

Although skin thickness is a relatively minor consideration in breast dosimetry with regards the mammography, the skin surrounding the breast tissue acts like an X-ray beam filter that is preferentially removing low-energy X-ray photons which contribute primarily to radiation dose to the breast (3). Huang etal. (3) have investigated the effect of breast skin thickness on mammographic dosimetry in terms of normalized glandular dose $\left(\mathrm{D}_{\mathrm{gN}}\right)$ coefficients. They reported the mean breast skin thickness as $1.45 \pm 0.30 \mathrm{~mm}$. Although they did not quantify the thickness variation with age, the reported mean thickness was in accordance with ref, (2) and contrary with some previously published works (4-6) that performed their study based on the assumption of a 4-mm breast skin thickness. It is interesting to know that the mammography images obtained by X-ray techniques are further processed by using various optical techniques like the ones presented in $(7,8)$.

The aim of this work is to use the Monte Carlo technique to assess the mammographic X-ray spectra in terms of transmission factor and its variation by the age-related changes in the breast skin thickness, for the X-rays in the energy range of 15-35 $\mathrm{keV}$ (covering all the energy intervals proposed by different references ${ }^{(9-12)}$ for the X-rays in mammography).

There have been studies in recent years, investigating photon attenuation in the human body organs for different energy ranges of radiation and using different simulation codes (13-17). However, a wide energy range of X-rays has been considered in this study to simulate some attenuation characteristics of the body skin, besides the transmission factor of the breast skin which depends on the thickness. To date, no study was found that investigated both of these aspects in the low-energy X-ray range.

The MCNP6.2 (Monte Carlo N-Particle, version 6.2) code is used to perform the theoretical experiment. In order to validate the estimations of MCNP, the mass attenuation coefficients estimated by this code have been compared with XCOM calculations. XCOM is a program that can calculate the total mass attenuation of the photons with the desired energies from $1 \mathrm{keV}$ up to $100 \mathrm{GeV}$, through the arbitrary elements, compounds, or mixtures, by deterministic methods (18). This program is freely available on the National Institute of Standards and Technology (NIST) website (19).

After observing good agreement between the two methods (Monte Carlo and deterministic methods), other attenuation characteristics such as linear attenuation coefficient, mean free path (MFP), and half-value layer thickness (HVL) of the body skin were calculated as well. It is worth mentioning that this work has been done with a metrological approach. However, the results of the first part of the study (description of the transmission factor as a function of skin thickness (age)) can be used in biology-related fields regarding mammography and the results of the second part (description of the other attenuation parameters) can be useful in the field of the dosimetry and/or shielding against ionizing radiation.

\section{MATERIALS AND METHODS}

The transmission factor of the skin is a dimensionless parameter, depending on the thickness of the skin and the energy of the incident beam. The transmission factor is the only attenuation characteristic against X-ray photons which directly depends on the sample thickness. It is defined by equation (1) (20).

$T(E, x)=\frac{\Phi(E, x)}{\Phi(E, 0)}$

$\Phi(E, X)$ is the average flux in a detector for the photons with energy $E$, passing through a sample of thickness $x . \Phi(E, 0)$ is the average flux in the detector for the same photon energy, in the absence of the sample. According to equation (1), the transmission factor is an adequate parameter to describe the dependency of the $\mathrm{X}$-ray spectra on the sample thickness. Here, the sample is the breast skin which its transmission factor is described for photons of 15-35 keV. As stated before, some variations happen to the breast skin thickness as a woman advances in age and these variations affect the transmission factor of the breast skin against the

Int. J. Radiat. Res., Vol. 19 No. 4, October 2021 
mammographic X-rays.

Authors of the ref. (2), have carried out a study to quantify the effect of aging on breast skin thickness and elasticity. To this end, the left breast of 339 female participants classified into four age groups (18-24 years, 25-44 years, 45-64 years, and +65 years), was divided into four quadrants (superior, lateral, inferior, and medial quadrants, relative to the nipple). The skin image was captured utilizing a $20 \mathrm{MHz}$ ultrasound probe (SonoSite 180PLUS, SonoSite, Australia). Then the thickness of the dermal layer of the skin was measured from the ultrasound image using IMAGEJ software (National Institute for Health, Bethesda, USA). The results of their study are summarized in table 1.

Table 1. The average breast skin thickness $(\mathrm{mm})$ for each participant group, at four breast quadrants (2).

\begin{tabular}{|c|c|c|c|c|}
\hline \multirow{2}{*}{$\begin{array}{c}\text { Age groups } \\
\text { (years) }\end{array}$} & \multicolumn{4}{|c|}{ Breast skin thickness (mm) } \\
\cline { 2 - 5 } & Superior & Lateral & Inferior & Medial \\
\hline $18-24$ & 1.84 & 1.74 & 1.97 & 1.97 \\
\hline $25-44$ & 1.85 & 1.73 & 1.94 & 1.95 \\
\hline $45-64$ & 1.72 & 1.5 & 1.8 & 1.8 \\
\hline+65 & 1.67 & 1.38 & 1.81 & 1.81 \\
\hline
\end{tabular}

As is evident from table 1 , the breast skin thickness significantly decreases from 45 years of age onwards. It also can be observed that the thinnest and thickest parts of the breast skin are the lateral and the medial quadrants relative to the nipple, respectively. To assess the effect of breast skin thickness variation on the transmission factor, the data of table 1 for lateral and medial quadrants (as two extremes of breast skin thickness) have been applied as input and the transmission factor has been estimated using equation (1), for the aforementioned age groups. For this purpose, a theoretical experiment has been performed using the MCNP code that will be explained in Simulation with MCNP6.2.

In addition to the transmission factor, there are some other attenuation characteristics of the skin against the low-energy X-ray photons, independent of the thickness. Linear and Mass attenuation coefficients, MFP, and HVL are of those parameters. Although these parameters

Int. J. Radiat. Res., Vol. 19 No. 4, October 2021 are independent of the skin thickness, surveying them as a function of energy is useful for dosimetry/shielding purposes in the low-energy $\mathrm{X}$-ray region. The linear attenuation coefficient $(\mu)$, which is typically expressed in the unit of $\left(\mathrm{cm}^{-1}\right)$, has a logarithmic relationship with the incident photons $\left(N_{0}\right)$ and the transmitted ones $(N)$ as equation (6) (21).

$\mu=\frac{1}{x} \ln \left(\frac{N_{0}}{N}\right)$,

where $x$ is the thickness of the sample material. To overcome the dependency of the attenuation coefficient on the material, the linear attenuation coefficient is divided by the material density. This parameter is called the mass attenuation coefficient $\left(\int \rho\right)$ which is expressed by equation (3) in the unit of $\left(\mathrm{cm}^{2} / \mathrm{g}\right)(21)$.

$\mu_{\rho}=\frac{\mu}{\rho}$

The mean free path (MFP), which is the average distance between two successive interactions of photons in the sample, is calculated by equation (4) in the unit of $(\mathrm{cm})(21)$.

$M F P=\frac{1}{\mu}$

The half-value layer thickness ( $H V L)$ is the thickness of the attenuator that reduces the intensity of photons by half of its initial magnitude. It is defined by equation (5) in the unit of $(\mathrm{cm})(21)$.

$H V L=\frac{\ln (2)}{\mu}$

Using equations (2)-(5), these attenuation characteristics of the skin are calculated by applying the MCNP code and for low-energy $\mathrm{X}$-ray photons (15-35 keV).

\section{Simulation with MCNP6. 2}

MCNP (Los Alamos National Laboratory, United States $(22,23)$ ) is a general-purpose transport code, working based on the Monte Carlo technique, which can precisely simulate the geometry of the setup, source, and sample. One can define any kind of material for sample, detector, collimators, etc. It also covers a wide 
range of energy for radiation sources. Moreover, MCNP considers by default all the possible interaction types of radiation with matter (photoelectric effect, Compton scattering, pair production, etc.), depending on the radiation type and energy. Thus, MCNP is a reliable simulation code to simulate the attenuation of $\mathrm{X}$-rays in any sample, which in this case is the breast skin. The 6.2 version (MCNP6.2) has been used in this investigation to simulate the experimental setup.

The geometry setup is similar to the one represented in figure 1 of the ref. (24). This simulated setup comprises two collimators, a detection area (an arbitrary detector), and the skin sample placed between the two collimators. The source and detector collimators are cylinders made of lead, with outer and inner diameters of $12 \mathrm{~cm}$ and $3 \mathrm{~mm}$, respectively. A monoenergetic point source is located at the entrance of the source collimator. To have more photons impinging on the detection area, the beam direction was aligned with the collimators. It is worth mentioning that since $\int, \mu_{\rho}, M F P, H V L$, and $T(E, x)$ are relative parameters (depending on the presence or absence of the attenuator), some considerations will not affect the final results. The source type, detector type, and beam direction (isotropic or anisotropic) are of those considerations. The dimensions are as follows: source collimator's length $16 \mathrm{~cm}$, detector collimator's length $22 \mathrm{~cm}$, the distance between the two collimators $8 \mathrm{~cm}$, detector's length $11 \mathrm{~cm}$, and detector's diameter $6 \mathrm{~cm}$.

The skin samples are disk-shaped with different thicknesses taken from table 1, for lateral and medial quadrants of the breast and four age groups. The simulations have been performed for X-ray photons of $15-35 \mathrm{keV}$ energies. It means that the energy of the X-ray photons varies in each individual run, starting from $15 \mathrm{keV}$ with 5 and $2 \mathrm{keV}$ increments for transmission factor calculation (equation (1)) and the other parameters (equations (2-5)), respectively. F4 tally of MCNP6.2 has been used to obtain the photon flux over the detector cell. F4 is indeed a volume-based tally for track length estimate of the cell flux. This tally estimates the flux averaged over a cell in the unit of (particles $/ \mathrm{cm}^{2}$ ). The number of tracked photons (events) was considered as $10^{6}$ in each simulation process.

To estimate the transmission factor, after modeling the above-mentioned geometrical setup the code was run for specific energy of incident photons, first in the absence of the skin sample to obtain $\Phi(E, 0)$ as the output of the F8 tally. Then the simulation is repeated in the presence of the skin sample between source and detector collimators for each specific thickness of the breast skin. Note that the user of MCNP should define precisely the composition of each material used in the simulation. For skin as the most important material of this work, the composition (the elemental concentration as weight in \%, mass fraction) is taken from ref. (13) and presented in table 2 . The average density of the skin is considered as $1.1 \mathrm{~g} / \mathrm{cm}^{3}$.

Table 2. The elemental concentration of the human skin (13).

\begin{tabular}{|c|c|}
\hline Elemental components & $\begin{array}{c}\text { Concentration in the human } \\
\text { skin (weight in \%) }\end{array}$ \\
\hline $\mathrm{H}$ & 0.10058 \\
\hline $\mathrm{C}$ & 0.22825 \\
\hline $\mathrm{N}$ & 0.04642 \\
\hline $\mathrm{O}$ & 0.619 \\
\hline $\mathrm{Na}$ & 0.00007 \\
\hline $\mathrm{Mg}$ & 0.00006 \\
\hline $\mathrm{P}$ & 0.00033 \\
\hline $\mathrm{S}$ & 0.00159 \\
\hline $\mathrm{Cl}$ & 0.00267 \\
\hline $\mathrm{K}$ & 0.00085 \\
\hline $\mathrm{Ca}$ & 0.00015 \\
\hline $\mathrm{Fe}$ & 0.00001 \\
\hline $\mathrm{Zn}$ & 0.00001 \\
\hline
\end{tabular}

\section{Calculation with XCOM}

The purpose of using XCOM besides MCNP is to validate the output of the Monte Carlo model by a deterministic method (XCOM), for the estimation of mass attenuation coefficients of the skin. To apply XCOM, it is enough to insert the skin composition (table 2) as an input and determine the desired energy interval in which the software calculates the mass attenuation coefficients. Then XCOM returns the desired output in a table and/or a graph. In this work, the mass attenuation coefficient of the skin was obtained by this method for 11 discrete

Int. J. Radiat. Res., Vol. 19 No. 4, October 2021 
energies, meaning 15-35 keV with $2 \mathrm{keV}$ increments.

\section{RESULTS}

To calculate the transmission factor for skin thicknesses mentioned in table 1 , the theoretical experiment has been performed by MCNP for the X-rays of $15,20,25,30$, and $35 \mathrm{keV}$, using the geometrical setup explained in the previous section. Note that only the energies of the X-rays are important here and the point source can be of any type in the simulation. First, the photon flux over the detector cell was calculated using F4 tally in the absence of the skin sample, and for each aforementioned energy. Then the simulation was repeated for each energy, in the presence of a thin layer of skin as the sample. The thickness of the sample in each run was taken from table 1 . The transmission factor for each energy and each skin thickness has been obtained, using equation (1). The variation of transmission factor with skin thickness is plotted in figure 1. One can use the linear fitted graphs in this figure to obtain the transmission factor for an arbitrary skin thickness, and the applied X-ray energies.

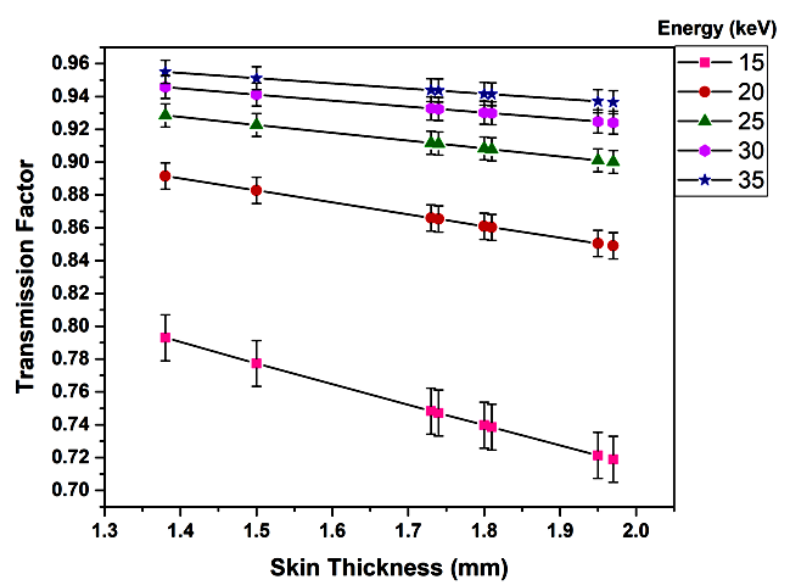

Figure 1. The transmission factor of the breast skin as a function of skin thickness and energy.

To have a better visualization, the transmission factor is also plotted as a function of age group (using data from table 1), for different photon energies. This is shown in figures 2(a) and 2(b) for the lateral and medial quadrants, respectively (as the thinnest and thickest parts of the breast skin).

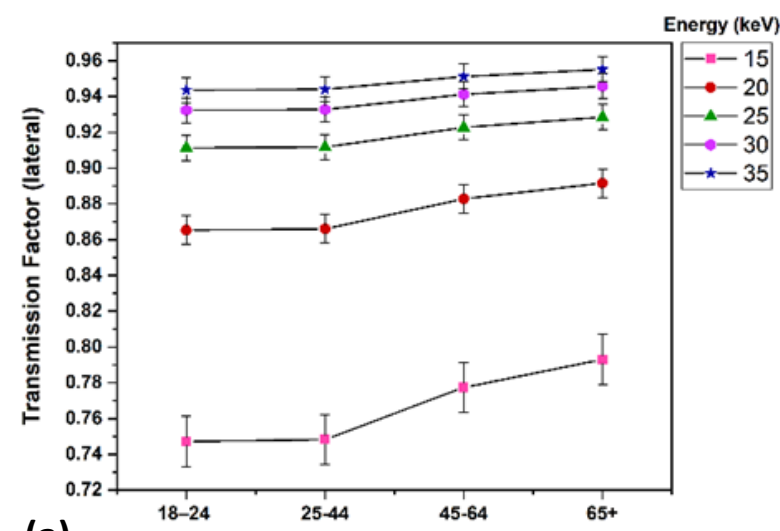

(a)

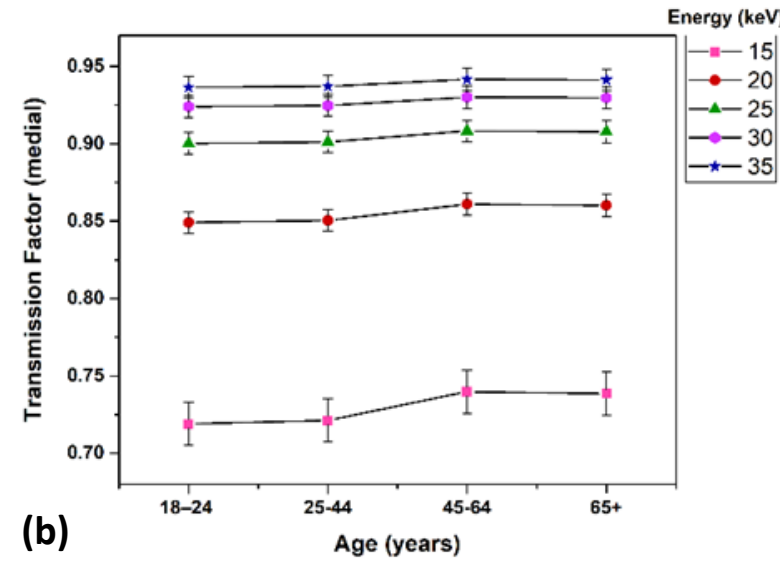

Figure 2. The transmission factor of the breast skin for four age groups and photon energies of 15, 20, 25, 30, and $35 \mathrm{keV}$

(a) lateral quadrant (b) medial quadrant.

To obtain the linear and the mass attenuation coefficients, the transmitted photon beam was estimated (using equations (2) and (3), respectively) for all the thicknesses of table 1 , and then the average values were calculated over those thicknesses. The variation of the linear attenuation coefficient of the skin with $\mathrm{X}$-ray energy is presented in figure 3.

The mass attenuation coefficients of the human skin, obtained by simulation and XCOM calculation are compared in figure 4. Equation (3) is used for the calculation based on MCNP output.

Table 3 shows the exact magnitude of photon mass attenuations of the skin, estimated by each method as well as the relative difference between them (MCNP and XCOM results) for each energy. 


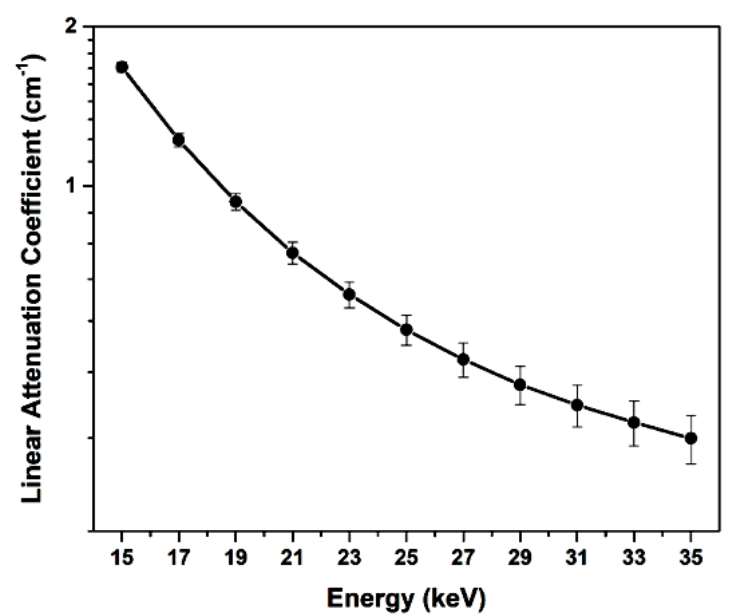

Figure 3. The linear attenuation coefficient of the human skin in the photon energy range of $15-35 \mathrm{keV}$, using Monte Carlo simulation MCNP6. 2 code and equation (2).

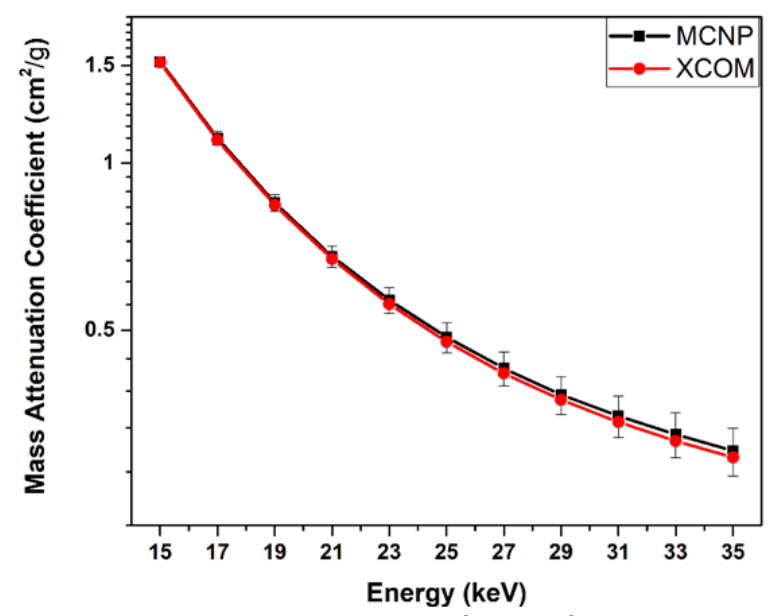

Figure 4. The mass attenuation coefficient of the human skin in the photon energy range of $15-35 \mathrm{keV}$, using Monte Carlo simulation MCNP6.2 code and XCOM program.

Table 3. Comparison of the results of MCNP and XCOM for mass attenuation coefficients of the low energy $\mathrm{X}$-rays through the human skin.

\begin{tabular}{|c|c|c|c|}
\hline \multirow{2}{*}{$\begin{array}{c}\text { X-ray } \\
\text { energy (keV) }\end{array}$} & \multicolumn{2}{|c|}{$\begin{array}{l}\text { Mass attenuation } \\
\text { coefficient }\left(\mathrm{cm}^{2} / \mathrm{g}\right)\end{array}$} & \multirow{2}{*}{$\begin{array}{c}\text { Relative } \\
\text { difference (\%) }\end{array}$} \\
\hline & MCNP6.2 & XCOM & \\
\hline 15 & 1.524 & 1.520 & 0.265 \\
\hline 17 & 1.110 & 1.100 & 0.891 \\
\hline 19 & 0.848 & 0.841 & 0.873 \\
\hline 21 & 0.679 & 0.672 & 1.114 \\
\hline 23 & 0.566 & 0.557 & 1.649 \\
\hline 25 & 0.486 & 0.477 & 1.905 \\
\hline 27 & 0.427 & 0.418 & 2.084 \\
\hline 29 & 0.382 & 0.374 & 2.137 \\
\hline 31 & 0.350 & 0.341 & 2.565 \\
\hline 33 & 0.325 & 0.316 & 2.862 \\
\hline 35 & 0.303 & 0.295 & 2.667 \\
\hline
\end{tabular}

The mean free path and half-value layer thickness of the human skin as a function of energy (equations 4 and 5, respectively) are shown in figure 5.

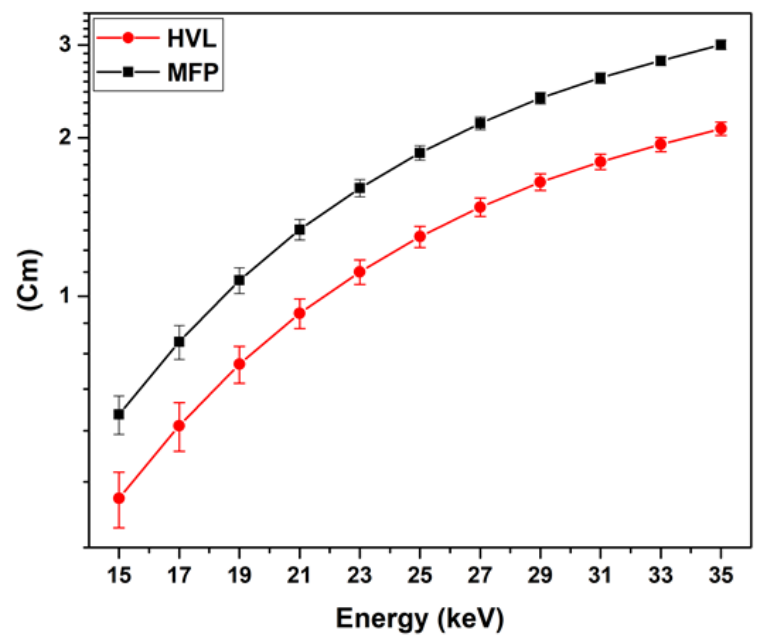

Figure 5. The mean free path (MFP) of the photons passing through the skin sample and the half-value layer (HVL) thickness of the human skin for the $\mathrm{X}$-ray photons as a function of energy, using Monte Carlo simulation MCNP6.2 code.

\section{DISCUSSION}

As is clear from figure 1, upon increasing the skin thickness, the transmission factor decreases linearly in the interested energy region, and it increases by increasing the photon energy. For low-energy photons, the difference between transmission factors is more noticeable, as the energy changes by $5 \mathrm{keV}$ increment. According to figure 1 and the data from table 1 , the transmission factor variation from the thinnest skin sample (lateral quadrant of the age group +65 ) to the thickest one (medial quadrant of the age group $18-24$ ) is $10.3 \%$, for the X-rays with $15 \mathrm{keV}$ energy. This variation decreases to $5.0 \%$, $3.14 \%, 2.35 \%$, and $1.98 \%$, respectively for 20 , 25,30 , and $35 \mathrm{keV}$ energies. In other words, increasing the X-ray energy leads to less sensitivity of the transmission factor to the skin thickness (age group). Although for other materials, the authors of ref. (20) have reported a similar behavior for the transmission factor, means being thickness- and energy-dependent and decreasing linearly by increasing the sample

Int. J. Radiat. Res., Vol. 19 No. 4, October 2021 
thickness.

Figure 2 has been provided for more visualization of the transmission factor as a function of age. It can be seen that the transmission factor is increasing by advancing age, but not linearly like figure 1 . Indeed, this variation gets more noticeable after 45 years. The older the patient, the more transmission factor of the breast skin against mammographic $\mathrm{X}$-rays. Thus, to have the best possible quality of $\mathrm{X}$-ray mammography images, the patient's age may also be taken into consideration as well as the other factors (25-28), when selecting the proper energies and for fine-tuning the used $\mathrm{X}$-ray generators.

Interpretation of figures 3-5 can provide information about the attenuation characteristics of very low-energy X-rays through the human skin. Figure 3 shows that the linear attenuation coefficient decreases more drastically, in the low-energy region. It means fine-tuning the energy should be performed more carefully in this region. In 2020, Soares et al. (29) performed an experimental study, using a similar set-up as the one simulated in this work, to measure the linear attenuation coefficient of the breast tissue against mammographic X-rays. Similar magnitude and behavior were reported by them for the linear attenuation coefficient of the breast tissue (even though not skin).

Figure 4 and table 3 represent the results of comparison between the Monte Carlo technique (MCNP) and the deterministic method (XCOM) for mass attenuation coefficients of the skin against X-rays of $15-35 \mathrm{keV}$ energy. This comparison shows a good agreement (especially in very low energy regions) and implies the reliability of the MCNP code to perform the theoretical experiment for attenuation characteristics calculations. Table 3 shows that the relative difference between the results of MCNP6.2 and XCOM varies from $0.2 \%$ up to $<3 \%$, for mass attenuation coefficients in the energy interval 15-35 keV. These differences for the X-rays with $15-21 \mathrm{keV}$ energy are less than $1 \%$ and for $21-35 \mathrm{keV}$ energy interval are between $1 \%$ and $3 \%$.

Similar studies have been carried out about the mass attenuation coefficients of the skin (13,
14). Although the authors of $(13,14)$ have performed their investigation for higher energies (60 keV- $2 \mathrm{MeV}$ ), their reported results of skin's mass attenuation coefficient show the same behavior as of the current study. This means the accordance between the results of this work with literature.

For the interested energy interval, the increase of MFP and HVL from the lowest energy $(15 \mathrm{keV})$ up to the highest energy (35 keV) is $2.52 \mathrm{~cm}$ and $1.75 \mathrm{~cm}$, respectively. When it comes to the low energy region, all the above-mentioned parameters show a drastic change even for a $2 \mathrm{keV}$ increment of energy. These findings are in accordance with refs. $(13,14$, $21,23,24,29)$, but for different energy intervals and sometimes different sample materials.

\section{CONCLUSION}

The skin thickness plays at least a minor role in breast dosimetry and there might be an assumption that the skin thickness variation with age is likely to affect the quality of the X-ray images, due to the photon attenuation in it. By achieving maximum visibility, the clinical staff can optimize the procedure of mammography and balance the quality requirements with the radiation dose to the patient, by choosing the most proper X-ray energy for each age group.

\section{Conflicts of interest: Declared none.}

\section{REFERENCES}

1. http://www.sprawls.org/resources/MAMMO/ (accessed April 2021)

2. Coltman CE, Steele JR, McGhee DE (2017) Effect of aging on breast skin thickness and elasticity: implications for breast support. Skin Res Technol, 23(3): 303-311.

3. Huang SY, Boone JM, Yang K, Kwan ALC, Packard NJ (2008) The effect of skin thickness determined using breast $C T$ on mammographic dosimetry. Med Phys, 35(4): 1199-1206.

4. Sarno $A$, Dance $D R$, van Engen RE, Young KC, Russo $P$, Lillo FDi, Mettivier G, Bliznakova K, Fei B, Sechopoulos I (2017) A Monte Carlo model for mean glandular dose evaluation in spot compression mammography. Med Phys, 44(7): 


\section{Zadehrafi / Breast skin thickness and mammographic X-ray}

3848-3860.

5. Sarno A, Mettivier G, Lillo FDi, Tucciariello RM, Bliznakova K, Russo P (2018) Normalized glandular dose coefficients in mammography, digital breast tomosynthesis and dedicated breast CT. Phys Med 55: 142-148.

6. Fedon C, Rigon L, Arfelli F, Dreossi D, Quai E, Tonutti M, Tromba G, Cova MA, Longo R (2018) Dose and diagnostic performance comparison between phase-contrast mammography with synchrotron radiation and digital mammography: a clinical study report. I Med Imaging, 5(1): 013503.

7. Ioan MR (2016) Investigation of RGB spectral components in the images captured through gamma rays affected optical focusing lens. Rom J Phys, 61(7-8): 1198-1206.

8. Ioan MR (2016) Study of the optical materials degradation caused by gamma radiation and the recovery process by controlled heat treatment. Rom J Phys, 61(5-6): 892-902.

9. Gennaro $G$ (2017) Clinical mammographic and tomosynthesis units. Handbook of X-ray imaging. Physics and Technology, 457.

10. Martini N, Koukou V, Michail C, Fountos G (2020) Dual energy $\mathrm{X}$-ray methods for the characterization, quantification and imaging of calcification minerals and masses in breast. Crystals, 10(3): 198.

11. Joe BN and Sickles EA (2014) The evolution of breast imaging: Past to Present. Radiology, 273(2): 23-44.

12. Lawaczeck R, Arkadiev VA, Diekmann F, Krumrey M (2005) Monochromatic X-rays in digital mammography. Invest Radiol, 40(1): 33-39.

13. Ermis EE, Pilicer FB, Pilicer E, Celiktas C (2016) A comprehensive study for mass attenuation coefficients of different parts of the human body through Monte Carlo methods. Nucl Sci Tech, 27: 54.

14. Tekin HO, Singh VP, Altunsoy EE, Manici T, Sayyed M (2017) Mass attenuation coefficients of human body organs using MCNPX Monte Carlo code. Iran J Med Phys 14: 229-240.

15. Salehi D, Sardari D, Jozani MS (2015) Investigation of some radiation shielding parameters in soft tissue. J Radiat Res Appl Sc, 8: 439-445.

16. Medhat ME, Shirmardi SP, Singh VP (2014) Comparison of Geant4, MCNP simulation codes of studying attenuation of gamma rays through biological materials with XCOM and experimental data. J Appl Computat Math, 3(6): 179.

17. Arslan H (2019) Photon attenuation parameters for some tissues from Geant4 simulation, theoretical calculations and experimental data: a comparative study. Nucl Sci Tech, 30: 96.

18. Berger MJ, Hubbell JH, Seltzer SM, Chang J, Coursey JS, Sukumar R, Zucker DS, Olsen K (last update: 2010) NIST Standard Reference Database 8 (XGAM). NIST, PML, Radiation Physics Division. Originally published as NBSIR 873597.

19. https://physics.nist.gov/PhysRefData/Xcom/html/ xcox1.html (accessed April 2021).

20. Bagheri R, Khorrami Moghaddam A, Yousefnia H (2017) Gamma ray shielding study of Bariume- Bismuthe- Borosilicate glasses as transparent shielding materials using MCNP-4C code, XCOM program, and available experimental data. Nucl Eng Technol, 49: 216-223.

21. Zadehrafi M, Olaru C, Ciobanu SA, Ormenisan GV (2020) Characteristics of lead glass for radiation protection purposes: A Monte Carlo study. Int J Radiat Res, 18(4): 907912.

22. Werner CJ, Bull JS, Solomon CJ, Brown FB, Mckinney GW, Rising ME, et al. (2018) MCNP6.2 Release Notes. Los Alamos National Laboratory, report LA-UR-18-20808.

23. Werner CJ (Ed.) (2017) MCNP user's manual - code version 6.2. Los Alamos National Laboratory, report LA-UR-1729981.

24. El-Khayatt AM, Ali AM, Singh VP, Badiger NM (2014) Determination of mass attenuation coefficient of low-z dosimetric materials. Radiat Eff Defect S, 169: 1038-1044.

25. Tomal A, Perez AMMM, Silva MC, Poletti ME (2015) Experimental evaluation of the image quality and dose in digital mammography: Influence of X-ray spectrum. Radiat Phys Chem, 116: 282-286.

26. Wong J, Xu T, Husain A, Le H, Molloi S (2004) Effect of area $X$-ray beam equalization on image quality and dose in digital mammography. Phys Med Biol, 49: 3539-57.

27. Pachoud M, Lepori D, Valley JF, Verdun FR (2004) A new test phantom with different breast tissue compositions for image quality assessment in conventional and digital mammography. Phys Med Biol, 49: 5267-81.

28. Cunha DM, Tomal A, Poletti ME (2012) Optimization of Xray spectra in digital mammography through Monte Carlo simulations. Phys Med Biol, 57: 1919-35.

29. Soares LDH, Gobo MSS, Poletti ME (2020) Measurement of the linear attenuation coefficient of breast tissues using polienergetic X-ray for energies from 12 to $50 \mathrm{keV}$ and a silicon dispersive detector. Radiat Phys Chem, 167: 108226. 